\title{
Consecutive sorting and phenotypic counting of CTCs by an optofluidic flow cytometer
}

\author{
Qingling $\mathrm{Li}^{1}$, Shuang $\mathrm{Cui}^{1}$, Yuehan $\mathrm{Xu}^{1}$, Yiguo Wang ${ }^{2}$, Feng $\mathrm{Jin}^{3}$, $\mathrm{Lu} \mathrm{Li}^{*}$ and Bo Tang*1 \\ ${ }^{1}$ College of Chemistry, Chemical Engineering and Materials Science, Key Laboratory of \\ Molecular and Nano Probes, Ministry of Education, Collaborative Innovation Center of \\ Functionalized Probes for Chemical Imaging in Universities of Shandong, Institute of Molecular \\ and Nano Science, Shandong Normal University, Jinan 250014, P. R. China \\ ${ }^{2}$ Shandong Provincial Qianfoshan Hospital, the First Hospital Affiliated with Shandong First \\ Medic al University, Jinan, 250014, P.R. China \\ ${ }^{3}$ Department of Thoracic Surgery, Shandong Provincial Chest Hospital Affiliated with Shandong \\ University, Jinan, 250013, P.R. China \\ *E-mail: tangb@sdnu.edu.cn; lilu5252@163.com
}




\section{Contents}

Fig. S1. Schematic construction and dimension of multistage microfluidic chip

Fig. S2. Simulation of three types of particles in the process of separation

Fig. S3. Simulation of matching unit of flow resistance

Fig. S4 Comparison of CellSearch and OFCM.

Table S1. Number of different CTC subpopulations in the 15 blood samples obtained from stage IV breast cancer patients.

Table S2. Number of different CTC subpopulations in the 31 blood samples obtained from stage I- IIIlung cancer patients.

Table S3. Number of different CTC subpopulations in the 41 blood samples obtained from stage IVlung cancer patients.

Video S1. A real-time movie showing the isolation of cancer cells from whole blood at the first stage outlet of the multistage microfluidic chip.

Video S2. A real-time movie showing the 3D focusing of cancer cells from whole blood at the second stage of the multistage microfluidic chip. 


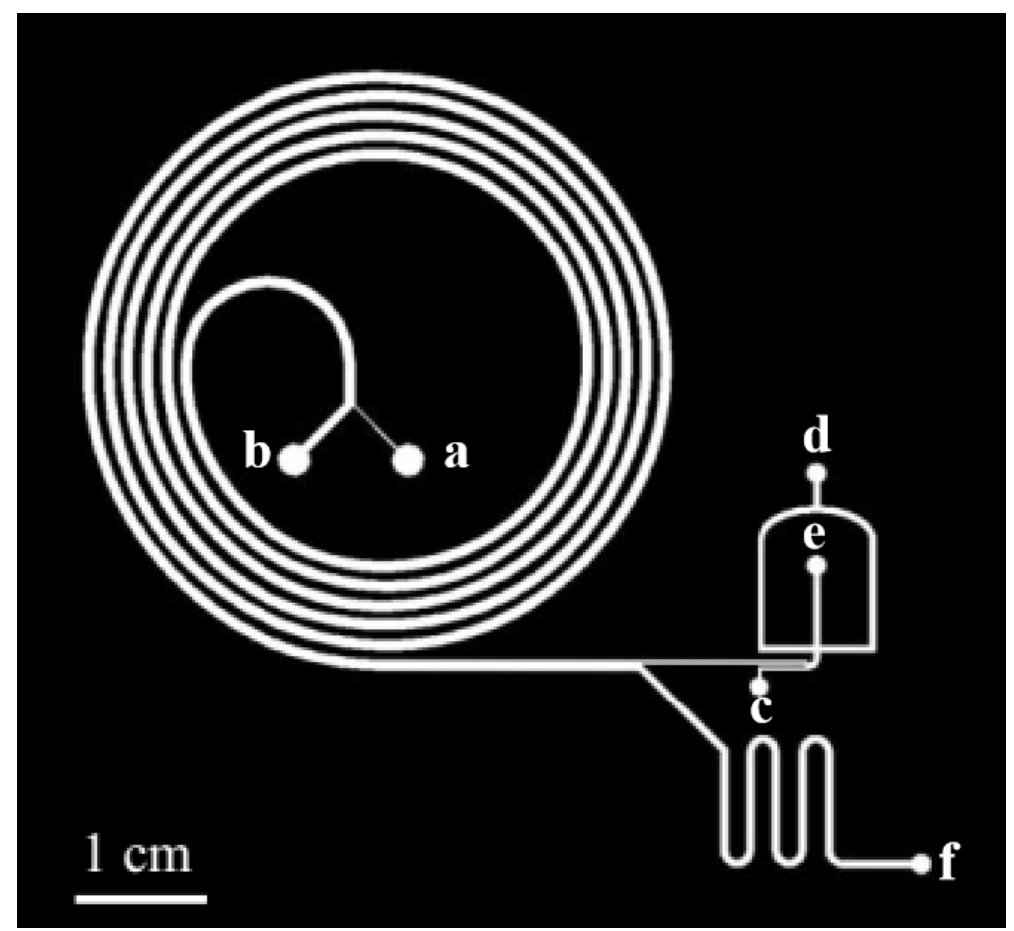

Fig. S1 Schematic construction and dimension of multistage microfluidic chip. The chip is $130 \mu \mathrm{m}$ high throughout. The first-stage separation structure employs 5 -spiral loops microchannel. The radius of the first curvature is $1 \mathrm{~cm}$ and the spacing between two adjacent loops is $500 \mu \mathrm{m}$. The width of inlet a microchannel is $75 \mu \mathrm{m}$. The width of inlet $\mathrm{b}$ microchannel is $425 \mu \mathrm{m}$. The second-stage 3D hydrodynamic focusing structure has a $90^{\circ}$ curved channel and the radius of the curve is $250 \mu \mathrm{m}$. The width of inlet $\mathrm{c}$ microchannel is $100 \mu \mathrm{m}$. The width of inlet $\mathrm{d}$ microchannel is $200 \mu \mathrm{m}$. The flow resistance matching part using a serpentine channel is $400 \mu \mathrm{m}$ width and the radius of the curve is $250 \mu \mathrm{m}$. The e and $\mathrm{f}$ is waste outlets. 

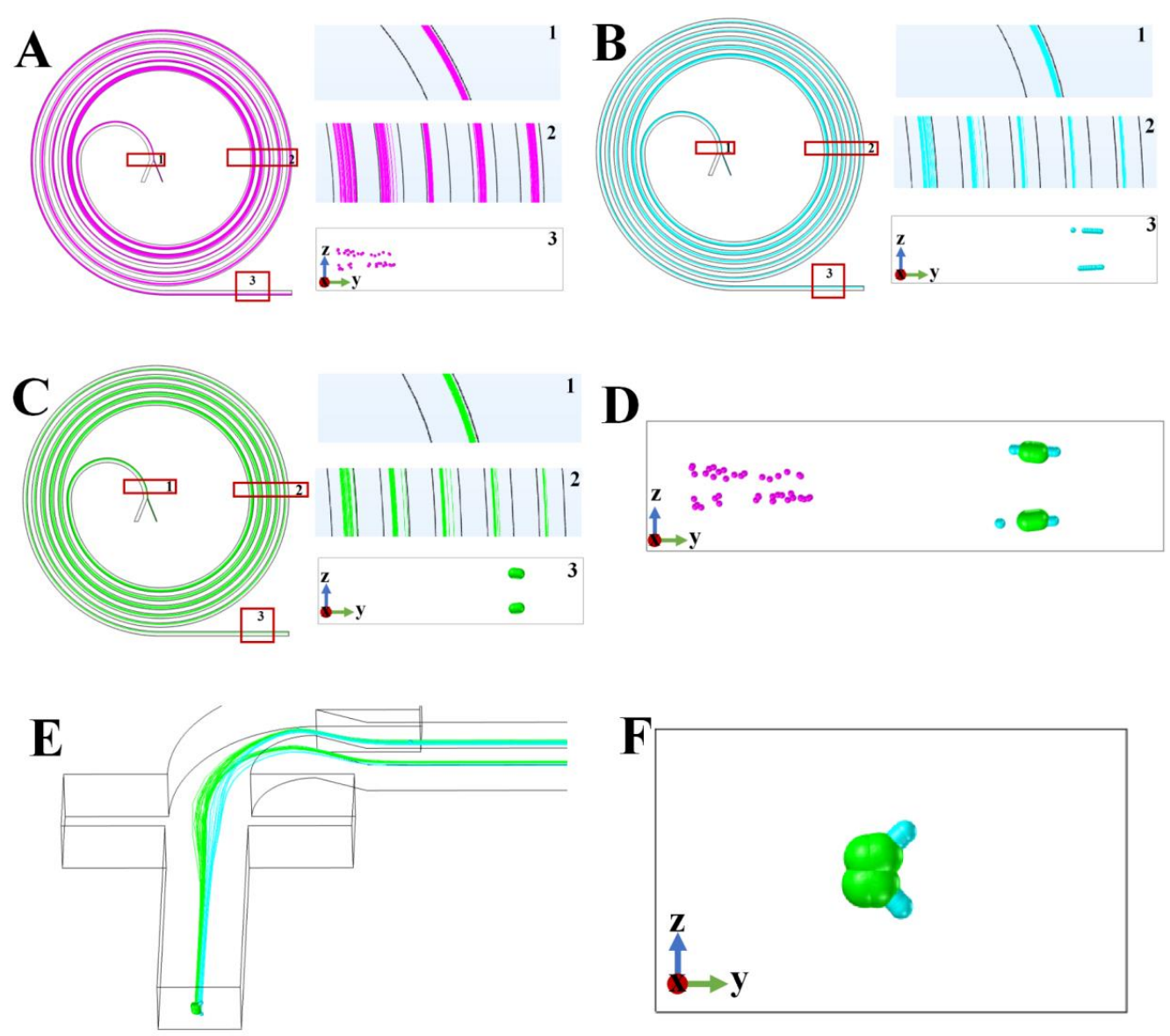

Fig. S2. Simulation of three types of in the process of separation. A, trajectories and position of $5 \mu \mathrm{m}$ particles at the exit. B, trajectories and position of $10 \mu \mathrm{m}$ particles at the exit. C, trajectories and position of $20 \mu \mathrm{m}$ particles at the exit. D, position of three types of particles at the exit. E, the simulation of $10 \mu \mathrm{m}$ and $20 \mu \mathrm{m}$ particles in the vertical direction and width direction. $\mathrm{F}$, the 3D focusing of particles on the cross section of the straight channel. 


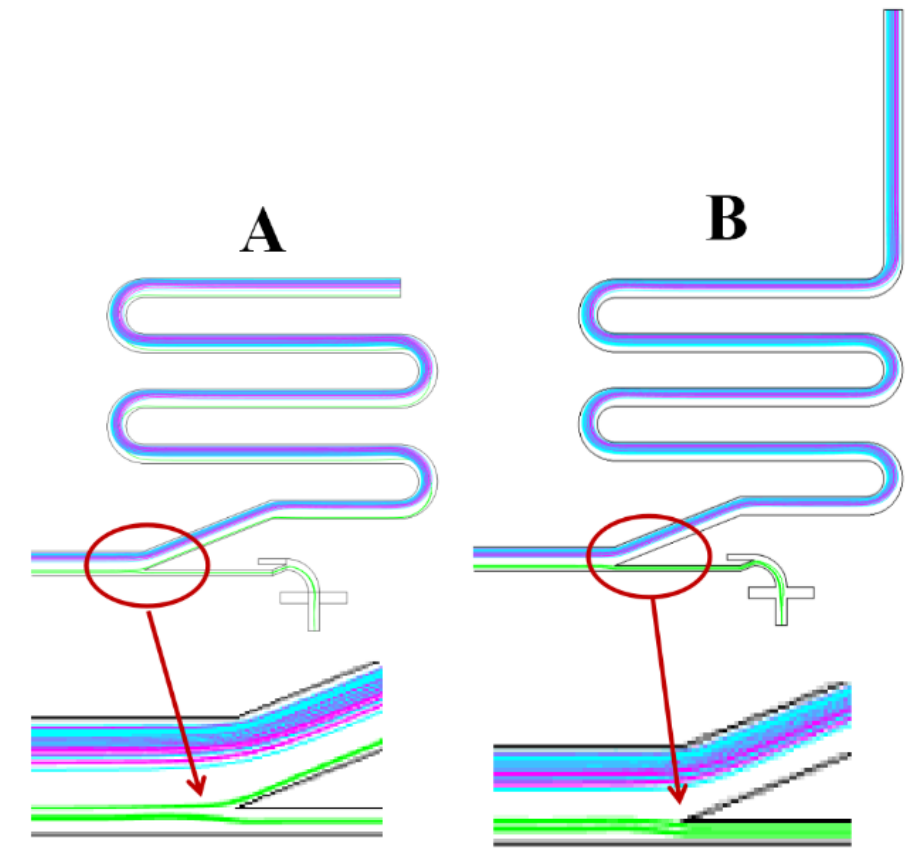

Fig. S3. Simulation of matching unit of flow resistance. A and B, the influence of flow resistance matching channel on separation effect. 

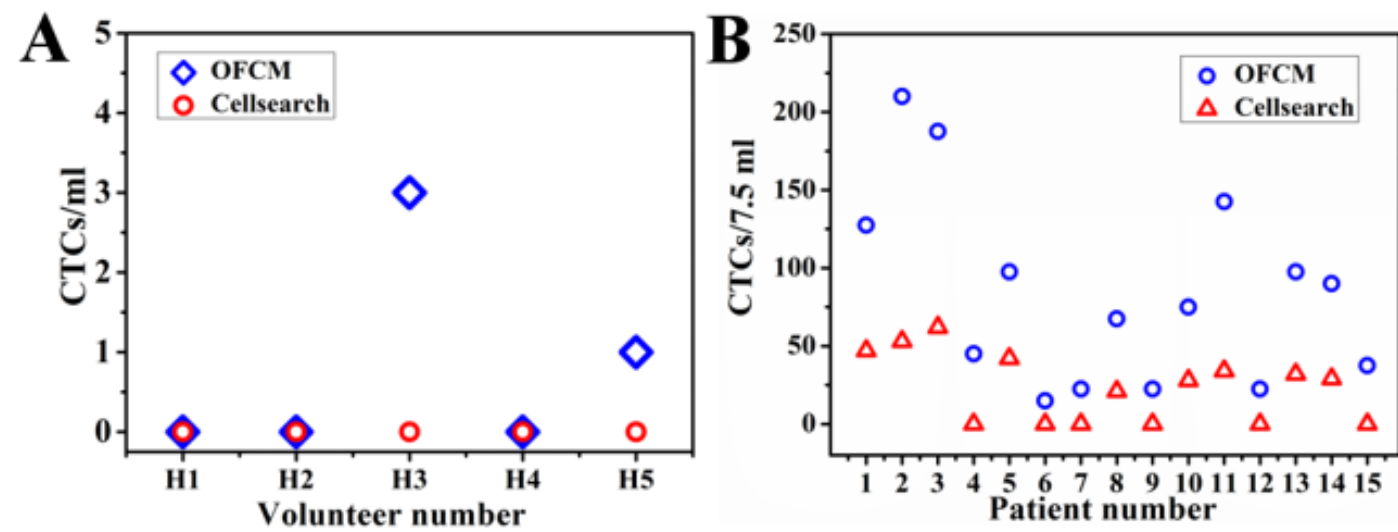

Fig. S4. Comparison of CellSearch and OFCM. A, 5 healthy volunteers samples ran with CellSearch and OFCM. B, 15 breast cancer patient samples ran with CellSearch and OFCM. 
Table S1. Number of different CTC subpopulations in the 15 blood samples obtained from stage IV breast cancer patients.

\begin{tabular}{|l|l|l|l|l|}
\hline Patient number & $\mathrm{E}^{+} \mathrm{CTC} / \mathrm{ml}$ & $\mathrm{M}^{+} \mathrm{CTCs} / \mathrm{ml}$ & $\mathrm{E}^{+} \mathrm{M}^{+} \mathrm{CTCs} / \mathrm{ml}$ & Total CTCs/ml \\
\hline 1 & 9 & 2 & 6 & 17 \\
\hline 2 & 13 & 8 & 7 & 28 \\
\hline 3 & 5 & 4 & 16 & 25 \\
\hline 4 & 2 & 1 & 3 & 6 \\
\hline 5 & 10 & 1 & 2 & 13 \\
\hline 6 & 1 & 1 & 0 & 2 \\
\hline 7 & 2 & 1 & 0 & 3 \\
\hline 8 & 5 & 3 & 1 & 9 \\
\hline 9 & 2 & 0 & 1 & 3 \\
\hline 10 & 5 & 1 & 4 & 10 \\
\hline 11 & 11 & 4 & 4 & 19 \\
\hline 12 & 2 & 0 & 1 & 3 \\
\hline 13 & 6 & 4 & 3 & 13 \\
\hline 14 & 5 & 2 & 5 & 12 \\
\hline 15 & 2 & 1 & 2 & 5 \\
\hline CTC Average & 5.3 & 2.2 & 3.7 & 11.2 \\
\hline Median & 5 & 1 & 3 & 10 \\
\hline
\end{tabular}


Table S2. Number of different CTC subpopulations in the 31 blood samples obtained from stage I- III lung cancer patients.

\begin{tabular}{|c|c|c|c|c|}
\hline Patient number & $\mathrm{E}^{+} \mathrm{CTCs} / \mathrm{ml}$ & $\mathrm{M}^{+} \mathrm{CTCs} / \mathrm{ml}$ & $\mathrm{E}^{+} \mathrm{M}^{+} \mathrm{CTCs} / \mathrm{ml}$ & Total CTCs/ml \\
\hline 1 & 8 & 5 & 11 & 24 \\
\hline 2 & 2 & 2 & 4 & 8 \\
\hline 3 & 5 & 2 & 3 & 10 \\
\hline 4 & 3 & 1 & 2 & 6 \\
\hline 5 & 4 & 4 & 14 & 22 \\
\hline 6 & 0 & 0 & 0 & 0 \\
\hline 7 & 4 & 2 & 2 & 8 \\
\hline 8 & 2 & 1 & 1 & 4 \\
\hline 9 & 1 & 0 & 0 & 1 \\
\hline 10 & 4 & 8 & 6 & 18 \\
\hline 11 & 0 & 0 & 0 & 0 \\
\hline 12 & 1 & 1 & 0 & 2 \\
\hline 13 & 0 & 1 & 3 & 4 \\
\hline 14 & 1 & 2 & 2 & 5 \\
\hline 15 & 4 & 3 & 4 & 11 \\
\hline 16 & 3 & 2 & 8 & 13 \\
\hline 17 & 9 & 5 & 5 & 19 \\
\hline 18 & 6 & 3 & 13 & 22 \\
\hline 19 & 1 & 1 & 0 & 2 \\
\hline 20 & 4 & 4 & 6 & 14 \\
\hline 21 & 1 & 2 & 1 & 4 \\
\hline 22 & 2 & 1 & 2 & 5 \\
\hline 23 & 1 & 0 & 2 & 3 \\
\hline 24 & 3 & 3 & 3 & 9 \\
\hline 25 & 2 & 1 & 2 & 5 \\
\hline 26 & 9 & 5 & 18 & 32 \\
\hline 27 & 20 & 11 & 10 & 41 \\
\hline 28 & 10 & 6 & 13 & 29 \\
\hline 29 & 4 & 3 & 2 & 9 \\
\hline 30 & 4 & 7 & 7 & 18 \\
\hline 31 & 2 & 2 & 7 & 11 \\
\hline CTC Average & 3.9 & 2.8 & 4.9 & 10.4 \\
\hline Median & 3 & 2 & 3 & 9 \\
\hline
\end{tabular}


Table S3. Number of different CTC subpopulations in the 41 blood samples obtained from stage IVlung cancer patients.

\begin{tabular}{|c|c|c|c|c|}
\hline Patient number & $\mathrm{E}^{+} \mathrm{CTCs} / \mathrm{ml}$ & $\mathrm{M}^{+} \mathrm{CTCs} / \mathrm{ml}$ & $\mathrm{E}^{+} \mathrm{M}^{+} \mathrm{CTCs} / \mathrm{ml}$ & Total CTCs/ml \\
\hline 1 & 3 & 12 & 4 & 19 \\
\hline 2 & 4 & 10 & 8 & 22 \\
\hline 3 & 8 & 2 & 13 & 23 \\
\hline 4 & 11 & 3 & 15 & 29 \\
\hline 5 & 12 & 3 & 16 & 31 \\
\hline 6 & 15 & 4 & 15 & 34 \\
\hline 7 & 7 & 2 & 11 & 20 \\
\hline 8 & 4 & 4 & 2 & 10 \\
\hline 9 & 25 & 9 & 2 & 36 \\
\hline 10 & 14 & 7 & 8 & 29 \\
\hline 11 & 16 & 8 & 2 & 26 \\
\hline 12 & 24 & 4 & 12 & 40 \\
\hline 13 & 17 & 17 & 9 & 43 \\
\hline 14 & 14 & 7 & 27 & 48 \\
\hline 15 & 16 & 11 & 26 & 53 \\
\hline 16 & 20 & 13 & 23 & 56 \\
\hline 17 & 21 & 14 & 27 & 62 \\
\hline 18 & 19 & 7 & 41 & 67 \\
\hline 19 & 34 & 18 & 22 & 74 \\
\hline 20 & 10 & 8 & 7 & 25 \\
\hline 21 & 45 & 4 & 27 & 76 \\
\hline 22 & 27 & 22 & 36 & 85 \\
\hline 23 & 41 & 24 & 38 & 103 \\
\hline 24 & 18 & 7 & 14 & 39 \\
\hline 25 & 26 & 10 & 54 & 90 \\
\hline 26 & 4 & 2 & 2 & 8 \\
\hline 27 & 5 & 3 & 3 & 11 \\
\hline 28 & 2 & 6 & 4 & 12 \\
\hline 29 & 3 & 5 & 7 & 15 \\
\hline 30 & 3 & 3 & 1 & 7 \\
\hline 31 & 9 & 4 & 12 & 25 \\
\hline 32 & 2 & 4 & 8 & 14 \\
\hline 33 & 6 & 7 & 3 & 16 \\
\hline 34 & 9 & 10 & 5 & 24 \\
\hline 35 & 7 & 4 & 3 & 14 \\
\hline 36 & 5 & 6 & 5 & 16 \\
\hline 37 & 12 & 5 & 7 & 24 \\
\hline
\end{tabular}




\begin{tabular}{|l|l|l|l|l|}
\hline 38 & 11 & 8 & 2 & 21 \\
\hline 39 & 7 & 8 & 4 & 19 \\
\hline 40 & 6 & 3 & 4 & 13 \\
\hline 41 & 11 & 2 & 5 & 18 \\
\hline CTC Average & 13.5 & 7.6 & 13 & 34.1 \\
\hline Median & 11 & 7 & 8 & 25 \\
\hline
\end{tabular}

